

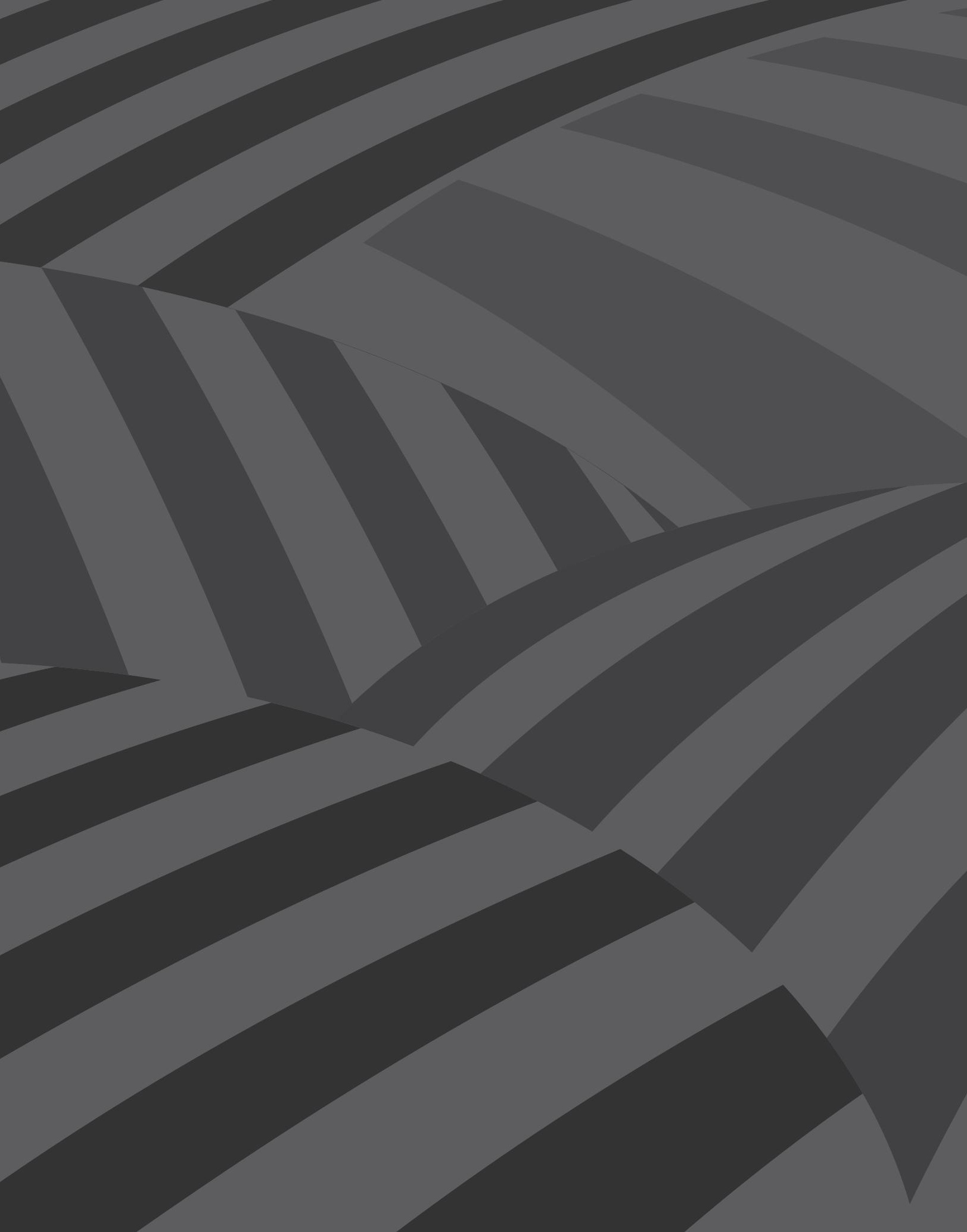




\title{
Derecho humano a la alimentación e igualdad en el tratamiento a los productores de alimentos, en los decretos expedidos en el marco del Estado de Emergencia Social, Económica y Ecológica del gobierno de Iván Duque
}

\author{
Por Paola Alejandra Galindo García*
}

Resumen: En el marco de las facultades otorgadas por la primera declaratoria de Estado de Emergencia Social, Económica y Ecológica en todo el territorio nacional (Decreto 417 de 2020), el gobierno de Iván Duque expidió, entre otros, el Decreto 486 de 2020, con la finalidad de proteger la logística relacionada con el abastecimiento alimentario durante la coyuntura de salud pública asociada a la pandemia de COVID-19. El Decreto 486 de 2020 es un instrumento normativo que operacionaliza la noción institucional de seguridad alimentaria y crea un incentivo económico para la producción de alimentos, en el contexto de la emergencia humanitaria y sanitaria. En el presente artículo se problematizan estas medidas para determinar si atienden al desarrollo del derecho humano a la alimentación y a la nutrición adecuadas, o si, por el contrario, se limitan a una versión restringida de la garantía de este, afectando con ello la posibilidad de justicia e igualdad material en tiempos de pandemia.

Palabras clave: derecho humano a la alimentación y nutrición adecuadas; seguridad alimentaria; economías campesinas, familiares y comunitarias; COVID-19.

* Filósofa de la Universidad Nacional de Colombia. Magister en Desarrollo Rural de la Pontificia Universidad Javeriana. Con experiencia en temas relacionados con conflictos agrarios y territoriales, derechos étnicos y campesinos, así como con modelos de ordenamiento territorial rural y urbano. Correo electrónico: pagalindog@ gmail.com 


\section{Human Right to Food and Equality in the Approach to Food Producers, in the Decrees Issued during Ivan Duque's government under the State of Economic, Social and Ecological Emergency}

Abstract: Within the framework of the powers granted by the first declaration of Social, Economic and Ecological Emergency made by the government of Iván Duque, Decree 486 of 2020 was issued with the purpose of protecting the logistics related to food supply during the public health situation associated with COVID-19. This decree is a regulatory instrument that operationalizes the institutional notion of food security, as well as the necessary incentives for food production in contexts of humanitarian and/or health emergency. This article problematizes these measures, indicating whether they are a development of the Human Right to Adequate Food and Nutrition or whether, on the contrary, they are limited to a restricted version of the guarantee of this right, thus affecting the possibility of justice and material equality in times of pandemic.

Keywords: Human Right to Adequate Food and Nutrition; Food Security; Peasant, Family and Community Economies; COVID-19.

Cómo citar este artículo: Galindo García, Paola Alejandra. (2021). Derecho humano a la alimentación e igualdad en el tratamiento a los productores de alimentos, en los decretos expedidos en el marco del Estado de Emergencia Social, Económica y Ecológica del gobierno de Iván Duque. Revista Controversia, 216, 169-200.

Fecha de recepción: 1 de noviembre de 2020

Fecha de aprobación: 17 de febrero de 2021

\section{Introducción}

$\mathrm{E}$

n los primeros días del mes de marzo de 2020, en que fue decretada la cuarentena en Colombia, la gente de las ciudades se aglomeró en las grandes plataformas comerciales en demanda de provisiones para su encierro, una hibernación masiva que exigía alimentos para varias semanas y todo lo necesario para afrontar los días de aislamiento impuestos por el gobierno nacional. Aunque la incertidumbre es cosa de todos los días, la pandemia le ha otorgado un brillo de excepcionalidad, animado por nuestras representaciones del apocalipsis y de las distopías contemporáneas. Lo que antes era una 
noticia distante, una enfermedad lejana e incomprensible, cambió con su llegada lo seguro, lo familiar, lo cotidiano.

Y lo cotidiano es también lo que comemos: qué comemos, cómo comemos, en qué lugar comemos. El tránsito de una vida volcada al afuera, que ocurría en las calles o en las oficinas, a una de reclusión en la que las prácticas del cuidado se traslaparon con los oficios y los horarios laborales, implicó, entre otras cosas, un retorno a la cocina. Pero no todas las personas contaban con alimentos en abundancia o suficientes; sin la aglomeración y la bulla del rebusque los hogares de trabajadoras y trabajadores informales, entre otros, se llenaron de banderas rojas, distintivo de la carencia de alimentos y evidencia de la desigualdad persistente expuesta, como nunca antes, por la pandemia. El pucho de dinero dejó de alcanzar y el hambre empezó a habitar con violencia las veinticuatro horas del día. Sin embargo, y a pesar de la magnitud del fenómeno, a la falta de víveres se la trató como un problema operativo, como si de llenar estómagos se tratara, en la suposición irresponsable que todo comestible es un alimento.

La respuesta institucional desconoció que el comer es una actividad inherente a la pervivencia y la dignidad de los seres humanos, enlazada a la práctica universal del cuido y, por tanto, proceso, nutrición, arraigo, memoria y vínculo. La alimentación fue drásticamente restringida a la logística del abastecimiento, a hacer llegar el volumen suficiente de alimentos a los centros urbanos, dentro de una idea de seguridad alimentaria circunscrita a la noción de disponibilidad.

Con esto en mente, el presente texto recoge algunas de las reflexiones surgidas en la Unidad de Trabajo Legislativo del senador Feliciano Valencia, de la cual hice parte, y fueron elaboradas y discutidas para ser empleadas en el ejercicio de control político, a propósito de la primera declaratoria del Estado de Emergencia Social, Económica y Ecológica. 
El objetivo de este texto es identificar y analizar algunas de las medidas implementadas por el gobierno nacional para proteger los sectores, y las acciones relacionadas con el abastecimiento alimentario durante la coyuntura de salud pública. En particular, se abordará lo relacionado con el Decreto 486 de 2020, por cuanto es uno de los instrumentos normativos que operacionaliza la versión institucional de seguridad alimentaria. Adicionalmente, y con el fin de problematizar este planteamiento normativo, tal medida será comparada con los componentes que definen el núcleo esencial del derecho humano a la alimentación y nutrición adecuadas, buscando precisar si existe una correspondencia entre las medidas adoptadas en los primeros meses del aislamiento obligatorio y el contenido del derecho mencionado. La evaluación de la correlación entre estos instrumentos permite determinar si las medidas implementadas por la institucionalidad, con relación al funcionamiento de los sistemas agroalimentarios, en un escenario de crisis sanitaria, garantizan o no el cumplimiento de este derecho a quienes son sus titulares.

\section{El derecho humano a la alimentación y nutrición adecuadas: un breve contexto}

Este es un derecho básico fundamental que, de acuerdo con la Observación General N. ${ }^{\circ} 12$ del Comité de Derechos Económicos, Sociales y Culturales (CDESC), es ejercido cuando "todo hombre, mujer o [niña/o], ya sea solo o en común con otros, tiene acceso físico y económico, en todo momento, a la alimentación adecuada o a medios para obtenerla" (1999, p. 2). ${ }^{1}$

De esta definición se desprenden cinco elementos que constituyen su contenido básico: (1) disponibilidad, (2) acceso, (3) calidad, (4)

1 En el ámbito internacional, la alimentación entendida como derecho humano ha sido reconocida también en el artículo 11 del Pacto Internacional de Derechos Económicos, Sociales y Culturales; en el artículo 25 de la Declaración Universal de Derechos Humanos; en el artículo 12 del Protocolo de San Salvador y en el artículo 25 de la Declaración Universal de Derechos Humanos. 
aceptabilidad y (5) sostenibilidad. En suma, esto quiere decir que los alimentos deben estar disponibles en la cantidad necesaria, de acuerdo con las necesidades asociadas a la ingesta calórica total para las personas y comunidades (Gómez et al., 2013). De acuerdo con la Food First Information and Action Network (FIAN, Colombia, 2015), la disponibilidad se logra mediante el autoabastecimiento, o gracias a su obtención a través de diferentes sistemas de producción, distribución, intercambio y comercialización; de ahí la importancia del reconocimiento de las economías campesinas y los sistemas tradicionales de producción.

Los alimentos deben estar en condición de accesibilidad física para disponer de ellos, con independencia de la capacidad adquisitiva; la ubicación geográfica; las situaciones de conflicto armado o de emergencia humanitaria; la pertenencia étnica, racial o de género; entre otras que puedan impedir a individuos, familias o colectividades acceder a ellos de manera autónoma, garantizando a su vez el goce o disfrute de otros derechos. En cuanto a la calidad, esta incluye condiciones como la variedad, diversidad, contenido nutricional y seguridad (Gómez et al., 2013).

El CDESC ha establecido que el cumplimiento de estos componentes implica "satisfacer las necesidades alimentarias de los individuos, sin sustancias nocivas y aceptables para una cultura determinada” (1999, p. 3). La variable cultural incorporada en esta última precisión refiere al criterio de aceptabilidad, de modo tal que la oferta alimentaria se corresponda cultural y nutricionalmente con las cosmovisiones de los pueblos y comunidades, teniendo en cuenta contextos ecosistémicos, formas propias de obtención, además de prácticas culinarias y gastronómicas. Finalmente, los sistemas agroalimentarios deben ser sostenibles, es decir, que todos los procesos, actores y relaciones que se articulan de manera dinámica al interior de estos interactúen con el ambiente garantizando la producción de alimentos a futuro.

Luego de que el CDESC adoptara la Observación General N. ${ }^{\circ} 12$ y aclarado su contenido normativo, precisó las obligaciones conexas de los 
Estados: respetar, proteger y realizar el derecho humano a la alimentación y nutrición adecuadas, lo que se traduce de manera operativa en (1) abstenerse de llevar a cabo acciones que tengan como resultado impedir la realización del derecho; (2) adoptar medidas para evitar que particulares priven del acceso a la alimentación a otros; y (3) promover la seguridad alimentaria entre la población. Que los Estados tengan estas responsabilidades configura, al tiempo, instrumentos y rutas para su exigibilidad desde las organizaciones sociales, los pueblos y comunidades (FIAN Colombia, 2015).

¿Por qué emplear como punto de referencia el derecho humano a la alimentación y nutrición adecuadas y no la categoría de seguridad alimentaria? Para dar cuenta de las razones que motivan este énfasis, resulta conveniente tener presentes los cambios en los paradigmas de desarrollo rural y las trasformaciones correspondientes de los sistemas agroalimentarios. En 1960, a propósito del rápido crecimiento poblacional y del aumento de las ayudas alimentarias en Asia, se reexaminó la noción de desarrollo vigente en la década anterior, según la cual el crecimiento económico consistía en un proceso de relocalización de los factores de producción de la agricultura a la industria, con un fuerte sesgo urbano (urban bias) dirigido a incentivar el crecimiento de las ciudades (Byerlee, Janvry y Sadoulet, 2009). En su lugar, se resalta el rol de la agricultura en el crecimiento económico, mediante la transformación de esta en un sector moderno gracias a la adopción de tecnologías.

Con la "crisis de escasez" ocurrida a mediados de 1970, la seguridad alimentaria empezó a ser relevante dentro de la agenda de los Estados. Esta fue entendida como "la capacidad de los países deficitarios de alcanzar en forma estable niveles de oferta interna que no fueran significativamente inferiores al nivel tendencial de la demanda efectiva" (Schejtman, 1988, p. 143). No obstante, y como lo ilustra este autor, en menos de una década se transitó de una escasez mundial de alimentos a una crisis de sobreoferta, gracias a los efectos de la implementación de la Revolución Verde, la cual "priorizó el desarrollo y la difusión de 
variedades de alto rendimiento de los principales cultivos básicos, en combinación con una utilización más intensiva de los insumos modernos como los fertilizantes inorgánicos y el riego" (Lipton y Longhurst, 1989 citado por Gómez et al., 2013, p. 10).

Tal situación contribuyó con la pérdida de relevancia de la seguridad alimentaria en las prioridades de la cooperación y de las políticas públicas del orden nacional.

Pese a ello, esta experiencia permitió construir la distinción entre disponibilidad (food avialiability) y acceso (acces to food), fundamental para los desarrollos en la década de 1980 del 'derecho a la alimentación’, pues la existencia de una oferta agregada de alimentos no asegura el acceso a estos, en particular a poblaciones que carecen de poder adquisitivo, generando manifestaciones de inseguridad alimentaria (Schejtman, 1988).

Esta limitación del concepto de seguridad alimentaria al de disponibilidad, desestima en su horizonte práctico situaciones como la escasez de alimentos a corto plazo, o producto de ciclos estacionales, marginación por condiciones de género o raza, o incluso fenómenos como el "hambre oculta” (Anderson, 2016).

Tal distinción fue pasada por alto en la crisis de alimentos de 2007 y 2008. Las trasformaciones de la agricultura, entre ellas la globalización, la emergencia de cadenas de suministro estrechamente coordinadas a gran escala, la demanda urbana de alimentos a bajo costo, los nuevos mercados para este sector, llevaron al posicionamiento de la tesis según la cual para evitar carestías y situaciones generalizadas de inseguridad alimentaria era necesario aumentar de manera masiva su producción, tanto así que

el mensaje que emergió de la Organización de las Naciones Unidas para la Alimentación y la Agricultura (FAO) (que muchos otros actores asumieron) fue que el mundo tenía que aumentar la producción alimentaria en un 
$70 \%$ (o más) para 2050, una afirmación que resta importancia al hecho de que durante la crisis de los precios de los alimentos había alimentos más que suficientes para satisfacer la demanda mundial, pero no había forma de proteger el acceso de las personas a esta oferta. (Murphy y Schiavoni, 2017, p. 21).

La distinción previamente enunciada pone de presente que las situaciones de malnutrición y desnutrición o de inseguridad alimentaria no ocurren necesariamente por desequilibrios entre la producción y la demanda de alimentos. La falta de acceso, por su parte, constituye una de las problemáticas sustantivas: esta se presenta bien por dificultades ocasionales, bien por restricciones estructurales producto de relaciones de desigualdad, las cuales configuran una brecha entre necesidades nutricionales e ingreso disponible para consumo alimentario en determinados sectores sociales (Schejtman, 1988, p. 142). Por estas razones:

Los activistas por la soberanía alimentaria insisten en que los y las proveedoras de alimentos a pequeña escala son los principales inversores en la producción de alimentos, y como tal merecen reconocimiento y apoyo. En cambio, el modelo de inversión con una mayor capitalización y políticamente más influyente implica enfoques más descendentes y centralizados, que a menudo se basan en partenariados público-privados (PPP) y suponen transferencias de tierras a gran escala. (Murphy y Schiavoni, 2017, p. 21).

De ahí que el enfoque de derechos humanos con respecto a la alimentación ponga al centro la necesidad de posicionar otro paradigma alimentario que proteja la producción agrícola local; las economías campesinas, familiares y comunitarias que la hacen posible; así como los sujetos asociados a estos sistemas de producción, a saber: el campesinado, así como los pueblos y comunidades étnicas desde una perspectiva de soberanía. Por lo anterior, las preguntas sobre dónde, cómo y quién produce los alimentos resultan necesarias cuando se hace referencia a este derecho fundamental. 


\section{Situación de la garantía del derecho humano a la alimentación y nutrición adecuadas en Colombia}

\subsection{Descripción del marco normativo vigente}

Para el caso colombiano, el derecho humano a la alimentación y nutrición adecuadas ha sido incorporado al sistema normativo a través de la ratificación de diferentes tratados internacionales, entre los cuales sobresalen el precitado Pacto Internacional de Derechos Económicos, Sociales y Culturales (1966); la Declaración Universal de los Derechos Humanos (1948); el Protocolo de San Salvador (1988); la Convención sobre la eliminación de todas las formas de discriminación contra la mujer (1979); la Convención sobre los Derechos del Niño (1989); y la Convención sobre los Derechos de las Personas con Discapacidad (2006). A nivel regional se ha dado un desarrollo normativo a través de instrumentos como el Protocolo Adicional de la Convención Americana sobre Derechos Humanos en materia de Derechos Económicos, Sociales y Culturales “Protocolo de San Salvador” (1988).

Por otro lado, en la Constitución Política de 1991, el artículo superior 65 otorga una especial protección estatal a la producción de alimentos. Así mismo, enfatiza en la necesidad de garantizar medidas diferenciales a ciertos colectivos sometidos a condiciones históricas de discriminación. En este sentido, el artículo 43 dispone que las mujeres gocen de un subsidio alimentario durante el embarazo, en los casos de desempleo o desamparo; el artículo 44 eleva a derecho fundamental de los niños y niñas la alimentación; y, finalmente, el artículo 46 consagra la protección reforzada a la tercera edad en materia alimentaria (Cepeda y Valencia, 2020).

Por su parte, la jurisprudencia constitucional ha reconocido que el derecho a una alimentación nutricional y culturalmente adecuada está vinculado con la garantía de los derechos a la vida, la dignidad humana, la salud y la integridad personal, especialmente en los casos en que consagra un deber de protección reforzado (Cepeda y Valencia, 2020). 
En la intervención ciudadana al Decreto 486 de 2020, realizada por los senadores Iván Cepeda y Feliciano Valencia, dirigida a la Corte Constitucional, se señala que la jurisprudencia ha expuesto desarrollos sobre el derecho a la alimentación que profundizan su protección y garantía. En la sentencia C-348 de 2012, la Corte explicó que el derecho a la alimentación no se reduce a la libre potestad de los pueblos de determinar sus procesos de producción de alimentos, sino que incluye la garantía que esos "procesos de producción permitan el respeto y la preservación de las comunidades y sus formas de producción artesanales y de pequeña escala, acorde con sus propias culturas y la diversidad de los modos campesinos y pesqueros".

\subsection{Sobre la situación de inseguridad alimentaria}

La protección de la producción de alimentos y de la logística relacionada con su abastecimiento fue central en las medidas de emergencia, a partir de la alerta sobre una posible agudización de la situación de desnutrición en el mundo, en particular, para personas y comunidades pobres que habitan áreas remotas; migrantes, trabajadores y trabajadoras informales; así como grupos sociales que se encuentran en áreas afectadas por crisis humanitarias o conflictos armados. Tal advertencia, adelantada por la FAO y por el Grupo de alto nivel de expertos en seguridad alimentaria y nutricional (High Level Panel of Experts -HLPE), responde a la consideración que entre 2016 y 2018 se reportaron 821 millones de personas desnutridas, la mayoría de las cuales se encontraban habitando países de bajos ingresos ubicados en el sur global (HLPE, 2020), cuyo riesgo y vulnerabilidad se acentúan en la coyuntura del virus SARS-CoV-2.

De acuerdo con el informe presentado por el HLPE, los efectos de la COVID-19 impactarán directa e indirectamente el ciclo agroalimentario nacional. Directamente, porque afectará los sistemas de producción y abastecimiento; indirectamente, por la disminución del ingreso monetario de las familias y de su capacidad adquisitiva, teniendo como efecto una reducción de los alimentos consumidos y de su calidad, lo que afecta desproporcionalmente a las mujeres, "puesto que a menudo 
comen menos o no comen cuando escasean los alimentos, a efectos de asegurar que el resto de los miembros de su familia puedan comer" (Murphy y Schiavoni, 2017). En este documento se identifican tres tipos de impactos a ser tenidos en cuenta por los y las tomadoras de decisiones: 1) en los sistemas de abastecimiento, en lo relacionado con las actividades de producción, distribución y comercialización; 2) en la demanda de alimentos, por la reducción de la capacidad adquisitiva; y 3) en el acceso, por la situación de confinamiento obligatorio y restricción de la movilidad.

En consecuencia, las recomendaciones de estos organismos multilaterales y de sus instancias consultivas están relacionadas con la mitigación de los impactos previamente enunciados. Conviene destacar algunas de las recomendaciones del HLPE (2020):

1. Priorizar, por parte de los Estados, las poblaciones más vulnerables a los impactos de la COVID-19, como los adultos mayores, los enfermos, la población desplazada y los pobres urbanos. Se destaca el rol específico de las mujeres en los sistemas de salud y abastecimiento, en condición de productoras de alimentos, procesadoras y cuidadoras.

2. Proveer asistencia esencial en el corto plazo y proteger los medios de subsistencia en un largo plazo.

3. Proteger las cadenas de abastecimiento de alimentos, en particular los ciclos cortos de comercialización, como mecanismo para evitar interrupciones en el movimiento, comercio e intercambio de alimentos, al tiempo que se garantiza su calidad y pertinencia para las poblaciones y comunidades que los consumen.

4. Proveer los insumos necesarios para la producción de alimentos, protegiendo e incrementando la producción local de estos, incluidas las huertas caseras, con el fin de fortalecer la resiliencia alimentaria, 
minimizar los desperdicios y evitar el acaparamiento, de tal manera que sea posible un acceso equitativo a la comida.

De lo anterior se sigue que, en términos de política pública para la atención de la COVID-19, sean necesarias acciones o intervenciones estatales para proteger las fases y componentes del proceso alimentario, como la producción local de alimentos asociada a las economías familiares, campesinas y comunitarias; su distribución, reduciendo intermediarios y sobrecostos; además del acceso y consumo de estos, prestando especial atención a las poblaciones más vulnerables.

De acuerdo con la Encuesta Nacional de Situación Nutricional-ENSIN, $2015,{ }^{2}$ el $54,2 \%$ de los hogares colombianos presenta inseguridad alimentaria en el hogar, es decir, " 1 de cada 2 hogares continúa en esta situación en razón a que persisten determinantes sociales y económicos que impiden el logro del derecho a la alimentación”. Tal situación es más traumática en la ruralidad, pues, según esta misma encuesta, la inseguridad alimentaria en el hogar en zonas rurales es 1,2 veces mayor que en las cabeceras municipales.

Los pueblos y comunidades étnicas presentan una situación de inseguridad alimentaria mucho más aguda. De acuerdo con las Bases del Plan Nacional de Desarrollo 2018-2022, la pobreza multidimensional en población indígena es 2,5 veces superior al total nacional (alcanza el $45,8 \%$ ) y en comunidades negras, afrodescendientes, raizales y palenqueras es 1,5 veces más alta (llega al 26,9\%). Así las cosas, durante el 2016, el 23,94\% de la población en condiciones de pobreza multidimensional pertenecía a un grupo étnico. Adicionalmente, la situación de confinamiento obligatorio, con ocasión de la emergencia sanitaria, pone en riesgo de sufrir inseguridad alimentaria y desnutrición a estas

2 En esta participaron: el Instituto Colombiano de Bienestar Familiar, el Ministerio de Salud y Protección Social, el Instituto Nacional de Salud, el Departamento Administrativo para la Prosperidad Social y la Universidad Nacional de Colombia. 
poblaciones, tanto más cuando en condiciones habituales el $77 \%$ de los hogares indígenas y el 68,9\% de los hogares afrodescendientes presentan inseguridad alimentaria y nutricional. Situación que se agudiza en niños y niñas, ya que el $29,6 \%$ de los menores de 5 años indígenas y el $7,2 \%$ de los menores de 5 años afrodescendientes presentaron desnutrición crónica, frente al $10 \%$ de los menores de 5 años sin pertenencia étnica (Cepeda y Valencia, 2020).

Esta situación de inseguridad alimentaria —empleando el criterio de la ENSIN 2015 - se asocia también a la informalidad y a los procesos de precarización del trabajo. En un escenario de aislamiento obligatorio, las personas y familias cuyo sustento proviene de un ingreso diario y que no cuentan con prestaciones sociales, son quienes están en mayor riesgo de no garantía del derecho humano a la alimentación y nutrición adecuadas. Para el caso colombiano, durante el trimestre diciembre 2019 - febrero de 2020 la proporción de ocupados informales correspondía al 47,9\%. ${ }^{3}$ Conviene mencionar que para el mes de julio de 2020 la tasa de desempleo se ubicó en 20,2\% , lo que significó un aumento de 9,5 puntos porcentuales frente al año anterior (10,7\%). Estos datos ponen de presente la magnitud de la actual crisis económica que revirtió el proceso de reducción de la pobreza, cuyo efecto fue el crecimiento de una clase media frágil, es decir, hogares con niveles de ingreso apenas superiores a la línea de pobreza, y que para el 2019 alcanzaban una suma de 2,5 millones (Universidad de los Andes, 2020).

De acuerdo con la Nota Macroeconómica N. ${ }^{\circ} 20$ de la Universidad de los Andes, la fragilidad de esta clase media es producto de la incidencia de la informalidad, cuyo efecto es una alta debilidad de las fuentes de ingresos de estos sectores ante cambios o choques económicos y la posibilidad de caer en situación de pobreza monetaria, puesto que:

3 https://www.dane.gov.co/files/investigaciones/boletines/ech/ech_informalidad/ bol_ech_informalidad_dic19_feb20.pdf 
El ingreso mediano mensual se desplazaría a niveles más cercanos a la línea de pobreza y se ubicaría muy por debajo de la línea de vulnerabilidad. Esto resultaría en un incremento de cerca de 15 puntos porcentuales adicionales de pobreza: 7,3 millones de personas más que en 2019, y una caída total en los ingresos mensuales de 4,8 billones de pesos. (2020, p. 3).

Como se mencionó en líneas anteriores, esta situación tiene un correlato en el acceso a los alimentos, dada la disminución de la capacidad económica de las familias para acceder a estos. Santiago Castaño, estudiante de doctorado y miembro del Grupo de Socioeconomía, Instituciones y Desarrollo de la Universidad Nacional de Colombia, advirtió en un conversatorio virtual adelantado por este grupo de investigación en el mes de marzo de 2020 que, producto de esta situación, las transferencias económicas existentes (Familias en Acción, Jóvenes en Acción, Programa Adulto Mayor) y las transferencias extraordinarias (Programa Ingreso Solidario) resultan insuficientes para cubrir los bienes y servicios necesarios durante la crisis.

Este panorama obliga a volver sobre la necesidad de medidas redistributivas que hagan posible un escenario de justicia material: en tiempos de pandemia, así como se debe garantizar el acceso universal a la salud, en lo inmediato debe considerarse una renta básica que incorpore la garantía del derecho humano a la alimentación y nutrición adecuadas, pues las medidas para la contención del virus no pueden admitir o coexistir con el hambre.

\section{El rol de las economías campesinas en la garantía del derecho humano a la alimentación y nutrición adecuadas}

¿Por qué la producción local asociada a las economías campesinas, familiares y comunitarias es una garantía para el abastecimiento de alimentos? 
Según la FAO (2014), existen más de 500 millones de explotaciones agrícolas familiares en el mundo, que suponen el $98 \%$ de todas las explotaciones agrícolas y son responsables de al menos el $56 \%$ de este tipo de producción. Tales explotaciones tienen un rol central en la provisión de los alimentos que son consumidos globalmente, en particular en los centros urbanos.

En Colombia las economías campesinas, familiares y comunitarias preservan la tendencia mundial, por cuanto "contribuyen con un poco más de la mitad de la producción agrícola, con cerca del $80 \%$ de la producción cafetera y con una tercera parte del valor de la producción pecuaria” (varias fuentes, citadas en Forero et al., 2013). Este dato es reafirmado por el Observatorio Rural de la Universidad de La Salle (2019), según el cual la agricultura familiar participa en un 63\% en el valor de la producción agrícola y en un $32 \%$ en la alimentación de los colombianos, dado el aumento en la importación de alimentos en que participa con un $29 \%$.

Lo dicho anteriormente es confirmado por el Procurador Delegado para Asuntos Ambientales y Agrarios, en su Memorando 005 del 27 de marzo de 2020, donde resalta que

la agricultura campesina, familiar y comunitaria-ACFC produce más del 70\% de los alimentos del país, aportan entre el 40 y el $60 \%$ del valor de la producción, y sus sistemas productivos cubren más del $60 \%$ de la superficie agropecuaria siendo el primer generador de empleo rural, además de tener un papel invaluable en términos de conservación de la agrobiodiversidad y como guardiana de las costumbres y tradiciones culturales.

Lo anterior, además de evidenciar la relevancia de este tipo de sistemas de producción y los actores a estos vinculados -el campesinado, así como los pueblos y comunidades étnicas-, pone de presente su eficiencia económica: genera más empleo por hectárea; mejora la seguridad alimentaria de la población; y disminuye el riesgo de inse- 
guridad alimentaria de los y las productoras campesinas, indígenas y afrodescendientes (Berry, 2013). De ahí que lo razonable en función de la protección de la producción de alimentos y de su acceso pase necesariamente por proteger, dinamizar e invertir en este tipo de economías, que efectivamente se constituyen en una garantía no solo de seguridad alimentaria, sino, de manera más integral, del cumplimiento del conjunto de componentes del derecho humano a la alimentación y nutrición adecuadas, bajo un paradigma de soberanía alimentaria.

\section{Medidas dictadas por el gobierno nacional para la protección del abastecimiento alimentario, durante la emergencia sanitaria producto de la COVID-19}

El gobierno nacional ha reconocido la importancia del abastecimiento de alimentos y de la seguridad alimentaria de los hogares colombianos en la coyuntura del COVID-19, lo que es evidente en el Decreto 457 de 2020, que contempla como una de las excepciones al aislamiento obligatorio el conjunto de actividades asociadas a la producción agropecuaria. ${ }^{4}$ Adicional a ello, en el marco de las facultades especiales que le otorga el Decreto 417 de 2020, mediante el cual se declaró el Estado de Emergencia Económica, Social y Ecológica, el presidente de la República expidió cuatro decretos relacionados con la producción agropecuaria, los cuales se relacionan en el cuadro 1.

El que incorpora el mayor número de medidas es el Decreto 486 de 2020, pues 1) crea un incentivo económico para productores mayores

4 “La cadena de siembra, cosecha, producción, embalaje, importación, exportación, transporte, almacenamiento, distribución y comercialización de semillas, insumos y productos agrícolas, piscícolas, pecuarios y agroquímicos -fertilizantes, plaguicidas, fungicidas, herbicidas-; productos agropecuarios, piscícolas y pecuarios, y alimentos para animales, mantenimiento de la sanidad animal, el funcionamiento de centros de procesamiento primario y secundario de alimentos, la operación de la infraestructura de comercialización, riego mayor y menor para el abastecimiento de agua poblacional y agrícola, y la asistencia técnica. Se garantizará la logística y el trasporte de las anteriores actividades" (artículo 3, numeral 11). 


\section{Cuadro 1. Decretos para el sector agropecuario expedidos en el marco del Estado de Emergencia Económica, Social y Ecológica}

\section{Decreto \\ Epígrafe 0 título \\ Fecha de \\ expedición \\ Tema}

"Por el cual se deroga el Titulo 9 de la Parte 13 del Libro 2 del Decreto 1071

Decreto 471 de 2020

Decreto 486 de 2020

Decreto 507 de 2020 de 2015, Decreto Único Reglamentario del Sector Administrativo Agropecuario, Pesquero y de Desarrollo Rural, en lo relacionado con la política de precios de insumos agropecuarios”.

"Por el cual se crea un incentivo económico para los trabajadores y productores del campo y se adoptan otras medidas para garantizar el permanente funcionamiento del sistema de abastecimiento de productos agropecuarios y seguridad alimentaria en todo el territorio nacional, dentro del Estado de Emergencia Económica, Social y Ecológica”.

Abastecimien-

25 de marzo de precios de insumos agrícolas.
Por el cual se adoptan medidas para favorecer el acceso de los hogares más vulnerables a los productos de la canasta básica, medicamentos y dispositivos médicos, en el marco de la Emergencia Económica, Social y Ecológica decretada mediante el Decreto 417 de 2020. to alimentario.

"Por el cual se modifica parcialmente

Decreto 523 de 2020 el Arancel de Aduanas en relación con la importación de materias primas como el maíz amarillo duro, el sorgo, la soya y la torta de soya”.
Eliminación

7 de abril de aranceles a cereales importados.

Fuente: elaboración propia. 
de 70 años que tengan confinamiento obligatorio y no estén amparados por ningún programa social o beneficio monetario del Estado; 2) faculta al Banco Agrario y al Fondo para el Financiamiento del Sector Agropecuario (FINAGRO), como administrador del Fondo Agropecuario de Garantías (FAG), para celebrar acuerdos de recuperación y pagos de cartera (condonación de intereses corrientes y de mora, además de quitas de capital $^{5}$ ) con los productores agropecuarios; 3) dispone que las líneas especiales de crédito que cree la Comisión Nacional de Crédito Agropecuario para los productores agropecuarios afectados por las causas que originaron la declaratoria de emergencia "podrán cubrir los costos y gastos financieros asociados a las operaciones de crédito Agropecuario" (art. 3) ; 4) habilita al Fondo de Solidaridad Agropecuario para que, según su disponibilidad de recursos, adquiera de los intermediarios financieros la cartera de los productores inmersos en distintas situaciones de crisis, una de las cuales es el Estado de Emergencia Económica, Social y Ecológica; y, finalmente, 5) el artículo 5 le permite al Ministerio de Agricultura y Desarrollo Rural (MADR),

contratar de manera directa, previa justificación técnica, la logística y actividades necesarias para garantizar la seguridad alimentaria y abastecimiento de productos e insumos agropecuarios en el territorio nacional, así como todo lo relacionado con el desarrollo de los apoyos e incentivos que requiera el sector, a través de las entidades u organizaciones que administren recursos parafiscales del sector agropecuario y con la sociedad fiduciaria del sector agropecuario.

Las medidas contempladas en el Decreto 486 de 2020 tienen como objeto garantizar el permanente funcionamiento del sistema de abastecimiento y seguridad alimentaria en todo el territorio nacional durante la emergencia económica, social y ambiental provocada por la pandemia.

5 Acuerdos entre el banco y sus clientes que buscan solucionar situaciones de impago. 
Ahora bien, para establecer si tales medidas satisfacen su finalidad, resulta necesario evaluar hasta qué punto:

- Cumplen con la totalidad de los componentes del derecho humano a la alimentación y nutrición adecuadas, pues desde la perspectiva de los derechos humanos este incorpora un conjunto de acciones que impiden que la alimentación, y en general el ciclo agroalimentario, sean interpretados de manera estrecha o restringida, impidiendo además su logro progresivo.

- Se corresponden con las orientaciones de la FAO y el HLPE.

- Y si, finalmente, el tiempo que lleva su implementación ha demostrado resultados favorables vs. el objeto del decreto.

De acuerdo con la parte motiva del decreto en comento, tanto el abastecimiento como la seguridad alimentaria son acciones que garantizan la suficiencia y accesibilidad de la población a los alimentos necesarios para su subsistencia.

Sin embargo, aunque incorpora de manera general los componentes de disponibilidad, acceso y sostenibilidad del derecho humano a la alimentación y nutrición adecuadas, deja de lado los de calidad y adaptabilidad, lo que implica una restricción de aquello que se entiende por abastecimiento alimentario y que debe ser garantizado desde una perspectiva de los derechos humanos.

Frente a lo dispuesto por el Decreto 486 de 2020, una de las primeras medidas fue la creación de un incentivo económico de 80000 pesos, dirigido a 500000 trabajadores y productores del campo mayores de 70 años. Este monto corresponde apenas al $8 \%$ de un salario mínimo mensual legal vigente, es decir, resulta insuficiente para mejorar la situación de vulnerabilidad de esta población, considerando además que solo 1 de cada 10 personas que habitan la ruralidad en Colombia ha cotizado 
para acceder al beneficio de pensión. De igual manera desconoce: las desigualdades de género relacionadas con la propiedad de la tierra; el acceso a bienes comunes para la producción campesina y familiar, así como a los mercados; y principalmente que son las mujeres quienes, además, soportan la economía del cuidado del hogar, en particular la siembra, la recolección, la preparación y distribución de los alimentos entre los miembros del núcleo familiar.

Aunque esta medida bien podría cumplir con el componente "acceso" del derecho humano a la alimentación y nutrición adecuadas, al ser un aporte monetario que le permite a la población dentro de los rangos etarios más vulnerables a la COVID-19 acceder a bienes y alimentos que no se producen intra-predialmente, no es una disposición clara por cuanto no establece su periodicidad (semanal, quincenal, mensual), ni cómo se hará llegar a la población beneficiaria, tanto más si esta se encuentra confinada en áreas rurales dispersas. De ahí que sea razonable afirmar que al ser un aporte tan bajo en dinero y sin una periodicidad establecida, no garantiza el acceso a los alimentos de manera suficiente, sostenible y adecuada.

Por otro lado, el decreto crea una serie de instrumentos financieros que pueden contribuir con el mejoramiento de las condiciones económicas de producción y el aprovisionamiento básico de alimentos, porque según señalan FINAGRO/Misión para la Transformación del Campo/DDRS (2014), "el ahorro, el crédito, las transferencias, los pagos y los seguros le permiten a los productores compensar efectos de choques adversos que pueden llegar a reducir sus ingresos y deteriorar su nivel de vida” (p. 1). De manera particular, el artículo 3 del Decreto 486 de 2020, relativo a las líneas especiales de crédito que se creen, otorga los mismos beneficios a pequeños, medianos y grandes productores.

Esta medida, aunque encomiable en su propósito general, omite la desigualdad existente entre los actores que beneficia (pequeños, medianos y grandes productores) y la cadena de valor del sector agropecuario. 
Por ejemplo, aunque la línea especial de crédito Colombia Agro Produce resulta llamativa por no estar atada a ciertos tipos de inversión (como es el caso de otras que solo se aprueban para inversión en maquinaria, agricultura por contrato, bioseguridad, etc.), y tiene una tasa anual que se ubica por debajo de la inflación para pequeños productores $(3,5 \%)$ y para medianos y grandes productores $(4,5 \%)$, no tiene en cuenta las barreras de acceso al crédito agropecuario por parte de los pequeños productores.

De acuerdo con el informe de FINAGRO/Misión para la Transformación del Campo/DDRs (2014), liderado por José Antonio Ocampo, una de las razones principales para que la banca no atienda a los pequeños productores y productoras familiares es que no son sujetos de crédito, debido a la ausencia de garantías y de la documentación requerida, la falta de historia crediticia y

adicional a lo anterior, los costos de transacción se han convertido en un factor determinante para limitar la demanda por recursos de crédito formal. Los costos de transacción evaluados como puntos adicionales a la tasa de interés alcanzan a ser del orden de $20 \%$ sobre el valor del crédito. (p. 24).

Dentro de estos gastos, según el informe acabado de citar, se encuentran los costos de viaje, tiempo de espera, recolección de documentos, pago de comisiones para llenar los formularios, pago de derechos notariales, apertura de cuentas de ahorro, llamadas telefónicas, entre otros. Todo lo anterior, sumado a la falta de información acerca del financiamiento y las opciones que la banca formal ofrece, lleva a que los productores busquen otras fuentes de fondeo, sin importar el pago de unas tasas mucho más elevadas.

Estas dificultades logísticas se incrementan en un contexto de aislamiento obligatorio y de restricción de la movilidad. Sin un mecanismo de focalización de la política, estos beneficios son cooptados y aprovechados por quienes pueden cumplir con las exigencias del sector financiero, es 
decir, por aquellos que tienen mayor capacidad de endeudamiento y que pueden hacer grandes inversiones de capital.

Esto se hizo evidente apenas se implementó el decreto objeto de estudio, situación que oportunamente denunció la Contraloría General de la República en el comunicado de prensa N. ${ }^{\circ} 55$ de 2020. De acuerdo con la Contralora Delegada para el Sector Agropecuario,

de los $\$ 226.000$ millones colocados hasta el 8 de abril, $\$ 213.566$ millones han ido a grandes comercializadores y agroindustriales, $\$ 8.300$ millones para medianos, y sólo $\$ 4.200$ millones para pequeños productores. El informe detalla que de los $\$ 226$ mil millones, $\$ 196.133$ millones se colocaron por rubros de comercialización y/o transformación, costos operativos y anticipos. (p. 2).

En suma, esta denuncia ilustra los efectos de la ausencia de un enfoque diferencial que pusiera en el centro a comunidades campesinas y étnicas productoras de alimentos, e impidiera tanto la concentración del crédito en medianos y grandes productores como su destinación no a la producción de alimentos sino otro tipo de actividades, que es justamente lo que preserva el riesgo de inseguridad alimentaria y de no garantía del derecho humano a la alimentación y nutrición adecuadas.

Finalmente, la última medida contemplada por el Decreto 487 de 2020, artículo 5, le permite al MADR

contratar de manera directa, previa justificación técnica, la logística y actividades necesarias para garantizar la seguridad alimentaria y abastecimiento de productos e insumos agropecuarios en el territorio nacional (...) a través de entidades $u$ organizaciones que administren recursos parafiscales del sector agropecuario.

La Ley 101 de 1993 (Ley General de Desarrollo Agropecuario y Pesquero) creó un régimen general de contribuciones parafiscales agrope- 
cuarias y pesqueras que son administradas por entidades privadas con ánimo de lucro, representativas de la actividad gravada, es decir, por los gremios. En la actualidad existen 16 cuotas de fomento, cada una con una entidad administradora, ${ }^{6}$ entre las cuales se encuentran la Federación Colombiana de Ganaderos (FEDEGAN) y los gremios afiliados a la Sociedad de Agricultores de Colombia (SAC).

De acuerdo con el MADR, el número de productores/contribuyentes que conforman las entidades administradoras de los Fondos Parafiscales del Sector Agropecuario asciende a un total de 583 321, como se ilustra en el cuadro 2. Este total presenta varias dificultades relacionadas con uno de los criterios subyacentes asociados a la concentración de los beneficios del decreto evaluado, esto es, el de la representación. En primer lugar, son datos que tienen entre cuatro y dos años de desactualización, considerando que no todos los fondos indican la fecha de reporte. Adicionalmente, varios de estos administradores advierten dificultades para determinar el número de productores/contribuyentes asociados al gremio, considerando la alta informalidad del sector. De igual manera, este dato no distingue cualitativamente entre los productores de cada uno de los sectores, lo cual hace imposible diferenciar entre pequeños, medianos o grandes. Cabe decir que el Censo Nacional Agropecuario (2014) reporta la existencia de 2,7 millones de productores; contrastando este dato con la información previamente expuesta, el total de productores agremiados en estos fondos apenas correspondería al 21,6\% , advirtiéndose una posible situación de subrepresentación. Si este es el caso, ¿no debería el gobierno nacional incluir criterios para la incorporación de productores que, al no estar agremiados, pueden encontrarse en situación de mayor vulnerabilidad frente a variaciones

6 Contribución cafetera (Federación Nacional de Cafeteros -FNCC), Cuota de fomento arrocero (FEDEARROZ), cerealista (FENALCE), cacaotero (FEDECACAO), algodonero (CONALGODÓN), panelero (DEFEPANELA), tabacalero (FEDETABACO), palmero (FEDEPALMA), hortifrutícola (ASOHOFRUCOL), leguminosas de grano (FENALCE), ganadero y lechero (FEDEGAN), avícola (FENAVI), porcícola (Porkcolombia), cauchero (Confederación Cauchera Colombiana), papa (FEDEPAPA), fríjol soya (FENALCE). 
o situaciones de emergencia que afectan la producción de alimentos como una pandemia?

Por otro lado, de acuerdo con la Encuesta de Cultura Política de 2019 (2020), que gracias a la sentencia STP 2028 de 2018 de la Corte Suprema de Justicia incluyó una caracterización de la población campesina en Colombia, de las 43156 personas encuestadas en 24 departamentos, 34056 se consideran campesinos y campesinas, de las cuales el 21,2\% pertenecen por lo menos a un grupo, organización o instancia, en su mayoría a una Junta de Acción Comunal o a organizaciones campesinas o asociaciones agropecuarias. Ahora bien, ¿cuántos campesinos y campesinas se encuentran incorporados efectivamente en los fondos referidos y son susceptibles de ser beneficiarios de estas políticas?

Es a este tipo de agremiaciones a las que terminan dirigiéndose la mayoría de los beneficios financieros, ante la ausencia de un criterio diferencial que involucre a quienes efectivamente garantizan la seguridad y la soberanía alimentaria.

En consecuencia, las economías campesinas, familiares y comunitarias están lejos de ser beneficiadas por el esquema de compra de alimentos mediante contratación directa. Esta situación contraría lo recomendado por la FAO y en general por el sentido común respecto a las adquisiciones públicas de alimentos para fortalecer los mercados locales, conectar productores locales y consumidores, mejorar el contenido nutricional de los alimentos ofertados e incorporados a programas sociales del gobierno - Programa de Alimentación Escolar (PAE), Instituto Colombiano de Bienestar Familiar (ICBF), Unidad de Servicios Penitenciarios y Carcelarios (USPEC) y Hospitales Públicos-, asegurando al tiempo el acceso y control a recursos productivos, y precios justos y estables al campesinado, así como salarios dignos para quienes son trabajadores o proletarios del campo. 


\section{Cuadro 2. Total de productores/contribuyentes de los Administradores de la cuota de Fomento de los Fondos Parafiscales del Sector Agropecuario}

\begin{tabular}{|c|c|c|c|c|}
\hline $\begin{array}{l}\text { Adminis- } \\
\text { trador de } \\
\text { la cuota de } \\
\text { fomento }\end{array}$ & $\begin{array}{l}\text { Características } \\
\text { de los } \\
\text { contribuyentes }\end{array}$ & $\begin{array}{l}\text { Número de } \\
\text { contribu- } \\
\text { yentes }\end{array}$ & $\begin{array}{l}\text { Fecha } \\
\text { del } \\
\text { reporte }\end{array}$ & $\begin{array}{l}\text { Presenta dificultades } \\
\text { para determinar } \\
\text { la cantidad de pro- } \\
\text { ductores/contribu- } \\
\text { yentes por informa- } \\
\text { lidad del sector }\end{array}$ \\
\hline FNCC & & 541.182 & 2018 & No reporta \\
\hline FEDEARROZ & & 16.378 & 2016 & No reporta \\
\hline FENALCE & & 780 & 2018 & Sí \\
\hline FEDECACAO & $\begin{array}{l}\text { Compañía Nacional } \\
\text { de Chocolates y } \\
\text { Casa Luker repre- } \\
\text { sentan el } 85 \% \text { del } \\
\text { recaudo }\end{array}$ & 35 & $\mathrm{~N} / \mathrm{A}$ & No reporta \\
\hline CONALGODON & & 564 & 2018 & No reporta \\
\hline FEDEPANELA & & 6.397 & $\mathrm{~N} / \mathrm{A}$ & No reporta \\
\hline FEDETABACO & & 5.579 & $\mathrm{~N} / \mathrm{A}$ & No reporta \\
\hline FEDEPALMA & Plantas de beneficio & 90 & 2018 & No reporta \\
\hline ASOFROHUCOL & & 1.463 & 2018 & Sí \\
\hline FENALCE & & 433 & 2018 & Sí \\
\hline \multirow[b]{2}{*}{ FEDEGAN } & Plantas de beneficio & 225 & $\mathrm{~N} / \mathrm{A}$ & No reporta \\
\hline & $\begin{array}{l}\text { cooperativas, } \\
\text { plantas de acopio, } \\
\text { particulares }\end{array}$ & 113 & $\mathrm{~N} / \mathrm{A}$ & No reporta \\
\hline \multirow{2}{*}{ FENAVI } & $\begin{array}{l}\text { Producción pollo } \\
\text { de engorde }\end{array}$ & 2.347 & 2018 & No reporta \\
\hline & $\begin{array}{l}\text { Producción pollita } \\
\text { postura }\end{array}$ & 2.406 & 2018 & No reporta \\
\hline PORKCOLOMBIA & & 4.363 & 2018 & No reporta \\
\hline $\mathrm{CCC}$ & & 23 & $\mathrm{~N} / \mathrm{A}$ & No reporta \\
\hline FEDEPAPA & & 900 & 2018 & Sí \\
\hline FENALCE & & 43 & 2018 & Sí \\
\hline TOTAL & & 583.321 & & \\
\hline
\end{tabular}

Fuente: elaboración propia. 
De acuerdo con la FAO, el mercado especializado en atender las necesidades de compra de las Entidades Estatales en Colombia está avaluado en cerca de 2,5 billones de pesos anuales. La cifra se divide entre el Ministerio de Educación, con el Programa de Alimentación Escolar (PAE), los programas del ICBF y el sistema penitenciario y carcelario. También hacen parte del listado las Fuerzas Armadas, programas departamentales, hospitales, universidades y, en general, todas las entidades que se financian de recursos públicos para la compra de alimentos. (Citado en Prieto, 2020).

Gran parte de estos recursos podrían destinarse, vía contratación directa con organizaciones campesinas y étnicas, a respaldar el abastecimiento, aprovechando además las capacidades adquiridas en la producción y transformación de productos agropecuarios, en el marco de la implementación de los puntos 1 y 4 del Acuerdo de Paz, es decir, respaldar las iniciativas productivas relacionadas con los Programas de Desarrollo con Enfoque Territorial (PDET), el Programa Nacional Integral de Sustitución de Cultivos Ilícitos (PNIS), los proyectos productivos de los espacios territoriales de capacitación y reconciliación (ETCR), entre otras figuras de desarrollo territorial con enfoques productivo y de género.

\section{Reflexiones finales}

Frente a este escenario, resulta fundamental preguntarse qué tipo de redes respaldan la realización del derecho humano a la alimentación y nutrición adecuadas en la presente coyuntura pandémica. La respuesta evidente en función de lo expuesto es sin duda: las redes que hacen parte o constituyen la economía campesina, familiar y comunitaria. Siendo este el caso, no era necesario un instrumento normativo adicional para garantizar el abastecimiento alimentario, ya que tanto la Constitución como la Resolución 464 de 2017 "por la cual se adoptan lineamientos estratégicos de política pública para la Agricultura Campesina, Familiar y Comunitaria”, entre otros documentos de política pública, contemplan estrategias que podrían haber sido implementadas 
desde un criterio de justicia económica y de disminución de la desigualdad, con el fin de atender las necesidades alimentarias de las familias afectadas por una situación de pobreza monetaria, agudizada por las medidas implementadas para la contención del coronavirus.

De hecho, organizaciones campesinas y étnicas de todo el país señalaron la importancia de acoger las orientaciones de política existentes, ampliando de manera progresiva sus alcances, con el fin de potenciar acciones dirigidas al fortalecimiento de la oferta nacional de alimentos, la regulación y garantía de la comercialización de los alimentos de origen campesino, así como apoyos específicos a quienes vieron comprometidas sus cosechas por las afectaciones provocadas por la COVID-19. Lo anterior fue exhaustivamente expuesto en el componente de economía campesina, de la carta dirigida a Iván Duque por las organizaciones campesinas, bajo la consigna “iproteger al campesinado para proteger la vida!” (DeJusticia, 2020).

Por esta razón, la Red Nacional de Agricultura Familiar y las Unidades Técnicas Legislativas de varios congresistas de la bancada de oposición, en ejercicio del control político a los decretos expedidos en el marco del Estado de Emergencia Social, Económica y Ecológica, radicaron el 21 de julio de 2020 el Proyecto de ley 110 de 2020 "Por medio del cual se reforma el Decreto 486 de 2020 y se dictan otras disposiciones”. Tal proyecto pretende revertir los efectos regresivos del Decreto 486 de 2020 y, principalmente: (1) implementar transferencias monetarias a título de renta básica para los hogares en situación de pobreza y vulnerabilidad; (2) modificar la línea especial de crédito Colombia Agro Produce, dirigiéndola exclusivamente a pequeños productores de alimentos incorporando criterios que superen las barreras de acceso descritas en líneas anteriores; y (3) dirigir las compras directas de alimentos a organizaciones y productores de la economía campesina, familiar y comunitaria, y con ello el fortalecimiento de los ciclos cortos de comercialización, así como la promoción de entornos alimentarios saludables. 


\section{Referencias}

Anderson, Molly. (2016). Hacia un monitoreo del derecho a la alimentación y a la nutrición centrado en las personas. Observatorio del Derecho a la Alimentación y a la Nutrición, (8), 14-17.

Berry, Albert. (12 de marzo de 2013). 'Se necesita un cambio a favor de la pequeña agricultura'. El Tiempo. Recuperado de https://www.eltiempo.com/ archivo/documento/CMS-12681262.

Byerlee, Derek; Janvry, Alain de and Sadoulet, Elisabeth. (2009). Agriculture for Development: Toward a New Paradigm. Annual Review of Resource Economics, 1, 15-31. Recuperado de https://doi.org/https://doi.org/10.1146/ annurev.resource.050708.144239

Cepeda Castro, Iván y Valencia Medina, Feliciano. (2020). Intervención ciudadana Decreto legislativo 486 del 27 de marzo de 2020.

Comité de Derechos Económicos, Sociales y Culturales [CDESC]. (26 de abril a 14 de mayo de 1999). Observación General 12 del Comité de Derechos Económicos, Sociales y Culturales. El derecho a una alimentación adecuada (artículo 11): 12.05.99. Recuperado de https://www.minsalud.gov.co/ sites/rid/Lists/BibliotecaDigital/RIDE/INEC/IGUB/derecho-a-una-alimentacion-adecuada.pdf

Contraloría General de la República. (17 de abril de 2020). Comunicado de Prensa $N .^{\circ} 55$.

Corte Constitucional de Colombia. Sentencia C-348 de 2012 (M. P.: Jorge Ignacio Pretelt Chaljub; mayo 15 de 2012).

Decreto 417 de 2020 [Presidencia de la República]. Por el cual se declara un Estado de Emergencia Económica, Social y Ecológica en todo el territorio Nacional. DO N. ${ }^{\circ} 51259$.

Decreto 457 de 2020 [Presidencia de la República]. Por el cual se imparten instrucciones en virtud de la emergencia sanitaria generada por la pandemia del coronavirus COVID-19 y el mantenimiento del orden público. DO N. ${ }^{\circ}$ 51264. Recuperado de http://www.suin-juriscol.gov.co/viewDocument. asp?ruta $=$ Decretos $/ 30038972$ 
Decreto 486 de 2020 [Ministerio de Agricultura y Desarrollo Rural]. Por el cual se crea un incentivo económico para los trabajadores y productores del campo y se adoptan otras medidas para garantizar el permanente funcionamiento del sistema de abastecimiento de productos agropecuarios y seguridad alimentaria en todo el territorio nacional, dentro del Estado de Emergencia Económica, Social y Ecológica. Marzo 27 de 2020. DO N. ${ }^{\circ}$ 51.269. Recuperado de https://dapre.presidencia.gov.co/normativa/normativa/DECRETO 486 DEL 27 DE MARZO DE 2020.pdf

DeJusticia. (20 de abril de 2020). Llamado de las organizaciones campesinas a tomar medidas urgentes y necesarias en el marco del COVID-19. Recuperado de https://www.dejusticia.org/wp-content/uploads/2020/04/PROTEGER-AL-CAMPESINADO-PARA-PROTEGER-LA-VIDA-1.pdf

Fondo para el Financiamiento del Sector Agropecuario [FINAGRo], Equipo de la Misión para la Transformación del Campo y Dirección de Desarrollo Rural Sostenible [DDRs]. (Diciembre de 2014). Misión para la transformación del campo, sistema nacional de crédito agropecuario. Propuesta de reforma. Recuperado de https://colaboracion.dnp.gov.co/CDT/Agriculturapecuarioforestal y pesca/Sistema Crédito Agropecuario.pdf

Food First Information and Action Network [FIAN] Colombia. (2015). La exigibilidad del derecho humano a la alimentación. Qué es y cómo hacerla. Recuperado de https://www.fiancolombia.org/category/fian-nuestro-trabajo/ exigibilidad-del-derecho-a-la-alimentacion/

Forero, Jaime; Garay, Luis Jorge; Barberi, Fernando; Ramírez, Clara; Suárez, Myriam y Gómez, Ricardo. (2013). La eficiencia económica de los grandes, medianos y pequeños productores agrícolas colombianos. En Luis Jorge Garay, Reflexiones sobre la ruralidad y el territorio en Colombia. Problemáticas y retos actuales. Bogotá: Pontificia Universidad Javeriana/ Oxford Committee for Famine Relief.

Gómez, Miguel; Barret, Christopher; Raney, Terri; Pinstrup-Andersen, Per; Meerman, Janice; Croppenstedt, André; Lowder. Sarah,... Brian Thompson. (August, 2013). Post-Green Revolution food systems and the triple burden of malnutrition. ESA Working Paper, (13-02), Recuperado de http://www.fao.org/fileadmin/templates/esa/Papers_and_documents/ WP_13-02_Gomez_et_al.pdf 
High-Level Panel of Experts on Food Security and Nutrition [HLPE], (2020). Impact of COVID-19 on Food Security and Nutrition ( $f(n)$. Roma: High-Level Panel of Experts.

Instituto Colombiano de Bienestar Familiar [ICBF]. (2015). Encuesta Nacional de Situación Nutricional. Recuperado de https://www.icbf.gov.co/bienestar/ nutricion/encuesta-nacional-situacion-nutricional\#ensin3

Murphy, Sohia y Schiavoni, Christina. (2017). Diez años tras la crisis alimentaria mundial: afrontar el reto del derecho a la alimentación. Observatorio del Derecho a la Alimentación y a la Nutrición, (1), 18-31.

Observatorio Rural de la Universidad de la Salle. (2020). Definiciones, dimensiones y precisiones. Magazín Ruralidades y Territorialidades, 1(6), 7-18.

Organización de las Naciones Unidas para la Alimentación y la Agricultura [FAO]. (2014). Agricultores familiares. Alimentar el mundo, cuidar el planeta. Recuperado de http://www.fao.org/3/mj760s/mj760s.pdf

Prieto, Natalia. (4 de febrero de 2020). Ley de compras públicas: ies esta la oportunidad del campo? Semana Rural. Recuperado de https://semanarural.com/web/articulo/ley-de-compras-publicas-es-esta-la-oportunidad-delcampo-colombiano/1315

Procurador Delegado para Asuntos Ambientales y Agrarios. (27 de marzo de 2020). Memorando $N$. $^{\circ} 005$. Recuperado de https://es.calameo.com/ $\mathrm{read} / 0058735522 \mathrm{f} 37 \mathrm{e} 7 \mathrm{~b} 0 \mathrm{f} 29 \mathrm{~b}$

Schejtman, Alexander. (Diciembre de 1988). La seguridad alimentaria: tendencias e impacto de la crisis. Revista de la CEPAL, (36), 141163. Recuperado de https://repositorio.cepal.org/bitstream/handle/11362/11722/036141162_es.pdf?sequence $=1$ https://repositorio.cepal. org/bitstream/handle/11362/11722/036141162_es.pdf?sequence $=1$

Universidad de los Andes. (2020). Nota Macroeconómica N. ${ }^{\circ} 20$. Efectos en pobreza y desigualdad del COVID-19 en Colombia: un retroceso de dos décadas. https://uniandes.edu.co/sites/default/files/asset/document/notamacro20.pdf 